\title{
DARK DISCOLORATION OF THE DORSAL SKIN OF THE FINGER JOINTS \\ A POOR PROGNOSTIC SIGN IN RHEUMATOID ARTHRITIS
}

\author{
BY
}

\author{
J. L. KALLIOMÄKI
}

From the Department of Medicine, University Hospital, Turku, Finland

Pigmentary disturbances have long been regarded as skin manifestations sometimes seen in rheumatoid arthritis (Barber, 1938; Copeman, 1948). All rheumatologists may be familiar with the typical dermal sign in rheumatoid arthritis-dark discoloration of the dorsal skin of the finger joints. Lucherini and Cervini (1961) make special mention of this sign, using the name "melanosi del dorso delle dita".

In this paper I shall try to describe our experience of the clinical significance of this sign as I have not succeeded in finding reports of a similar kind in the literature.

\section{Material}

The series consists of 162 consecutive patients (124 women, $38 \mathrm{men}$ ) with rheumatoid arthritis seen in the Out-patients' Department for Rheumatoid Diseases in Turku. All these patients have been personally followed by the author, most of them for many years, and it has been possible to observe the clinical activity and course of disease and the response to therapy (gold, antimalarials, physical therapy).

\section{Results}

Most of the results are given in the Table (opposite) Dark discoloration of the dorsal skin of the finger $\stackrel{\omega}{N}$ joints was seen in 11.7 per cent. in this series of con-o secutive patients with rheumatoid arthritis (Figure)..

No significant difference was observed between females (frequency 10.5 per cent.) and males (frequency 15.8 per cent.), but the sign was noted among the patients with high clinical activity and poor therapeutic response much more frequently than expected $(P<0.01)$. Among the patients with an erythr8- $\vec{\theta}$ cyte sedimentation rate $\leqq 40 \mathrm{~mm}$./hr, the sign wơ less frequent than expected at the 5 per cenif: confidence level.

I have sometimes seen this sign in patients with disseminated lupus erythematosus if arthritic changes. are present in the finger joints, and also sometimes $\mathbb{\Phi}$ in patients with scleroderma or Raynaud's syndrome, $\stackrel{2}{\Rightarrow}$ but never in connexion with any other diseases.

As no histological or chemical examinations of the skin of this discoloration area have been per-

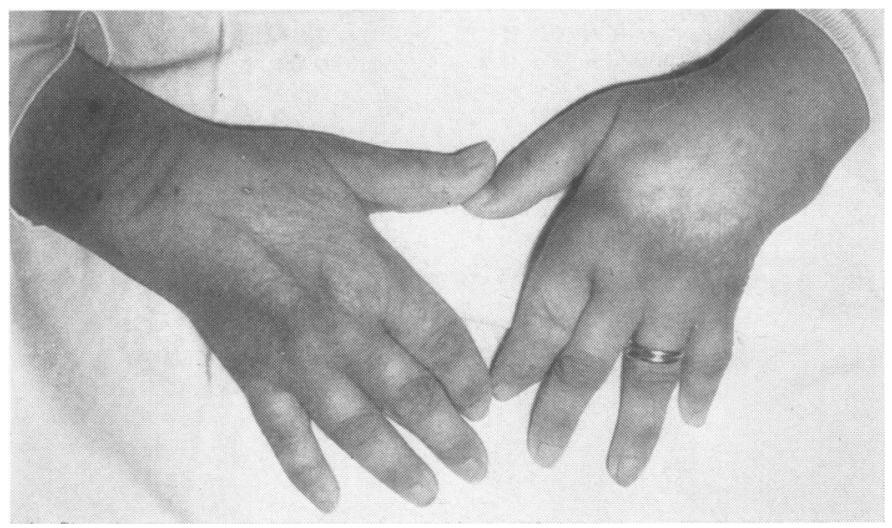

Figure.-A female patient with rheumatoid arthritis and typical discoloration of the dorsal skin of the finger joints. 
TABLE

DARK DISCOLORATION OF DORSAL SKIN OF FINGER JOINTS RELATED TO OTHER CLINICAL SIGNS

\begin{tabular}{|c|c|c|c|c|}
\hline \multirow{2}{*}{ No. of Cases } & \multirow{2}{*}{$\cdots$} & \multirow{2}{*}{ Total } & \multicolumn{2}{|c|}{$\begin{array}{c}\text { With Dark } \\
\text { Discoloration }\end{array}$} \\
\hline & & & No. & Per cent. \\
\hline Age (yrs) & $\begin{array}{l}\leqslant 20 \\
21-40 \\
\geqslant 41\end{array}$ & $\begin{array}{r}5 \\
32 \\
125\end{array}$ & $\begin{array}{r}2 \\
6 \\
11\end{array}$ & $\begin{array}{r}40 \cdot 0 \\
18 \cdot 8 \\
8 \cdot 8\end{array}$ \\
\hline $\begin{array}{l}\text { History } \\
\text { of Rheumatoid } \\
\text { Arthritis (yrs) }\end{array}$ & $\begin{array}{l}<1 \\
1-5 \\
\geqslant 6\end{array}$ & $\begin{array}{l}23 \\
51 \\
88\end{array}$ & $\begin{array}{l}4 \\
6 \\
9\end{array}$ & $\begin{array}{l}17 \cdot 4 \\
11 \cdot 7 \\
10 \cdot 2\end{array}$ \\
\hline $\mathrm{Hb}(\mathrm{g} . / 100 \mathrm{ml})$. & $\begin{array}{l}<11 \\
\geqslant 11\end{array}$ & $\begin{array}{r}27 \\
135\end{array}$ & $\begin{array}{r}5 \\
14\end{array}$ & $\begin{array}{l}18 \cdot 5 \\
10 \cdot 4\end{array}$ \\
\hline $\begin{array}{c}\text { Erythrocyte Sedimentation } \\
\text { Rate (mm./hr) }\end{array}$ & $\begin{array}{l}<40 \\
\geqslant 40\end{array}$ & $\begin{array}{r}55 \\
107\end{array}$ & $\begin{array}{r}13 \\
6\end{array}$ & $\begin{array}{r}23 \cdot 6 \\
5 \cdot 6\end{array}$ \\
\hline Clinical Activity* & $\begin{array}{r}0 \\
+ \\
++ \\
++\end{array}$ & $\begin{array}{l}22 \\
60 \\
55 \\
25\end{array}$ & $\begin{array}{r}0 \\
3 \\
4 \\
12\end{array}$ & $\begin{array}{r}0 \cdot 0 \\
5 \cdot 0 \\
7 \cdot 3 \\
44 \cdot 0\end{array}$ \\
\hline $\begin{array}{l}\text { Therapeutic } \\
\text { Response }\end{array}$ & $\begin{array}{l}\text { Good } \\
\text { Fair } \\
\text { Poor } \\
\text { Not } \\
\text { known }\end{array}$ & $\begin{array}{r}62 \\
51 \\
40 \\
9\end{array}$ & $\begin{array}{r}0 \\
2 \\
14 \\
3\end{array}$ & $\begin{array}{r}0.0 \\
4 \cdot 0 \\
35 \cdot 0 \\
33 \cdot 3\end{array}$ \\
\hline Total .. & $\cdots$ & 162 & 19 & $11 \cdot 7 \dagger$ \\
\hline
\end{tabular}

- 0 = Inactive.

$+=$ Slightly active.

$++=$ Moderately active.

$\dagger$ "Expected" frequency of dark discoloration in different clinical groups of rheumatoid arthritis.

formed, the pathogenesis of the sign cannot be discussed.
Summary

(1) Dark discoloration of the dorsal skin of the finger joints was seen in 11.7 per cent. of 162 consecutive patients with rheumatoid arthritis.

(2) High clinical activity and poor therapeutic response were characteristics of the patients with this sign.

\section{REFERENCES}

Barber, H. W. (1938). Proc. roy. Soc. Med., 31, 701.

Copeman, W. S. C. (1948). "Textbook of the Rheumatic Diseases." Livingstone, Edinburgh.

Lucherini, T., and Cervini, C. (1961). Acta rheumatologica (Geigy), Numero speciale, p. 42 (Symposium di studiosi italiani in campo reumatologico).

Coloration plus foncée de la peau dorsale des articulations des doigts: un mauvais signe pronostique dans l'arthrite rhumatismale

RÉSUMÉ

(1) Une coloration plus foncée de la peau dorsale des articulations des doigts fut observée dans $11,7 \%$ des 162 malades consécutifs atteints d'arthrite rhumatismale.

(2) Des poussées morbides prononcées et peu de succès thérapeutique caractérisaient les malades présentant ce signe.

Obscurecimiento de la piel dorsal de las articulaciones de los dedos: signo pronóstico desfavorable en la artritis reumatoide

Sumario

(1) Un obscurecimiento de la piel dorsal de las articulaciones de los dedos fué observado en un $11,7 \%$ de 162 enfermos consecutivos con artritis reumatoide.

(2) Un proceso mórbido pronunciado y poca respuesta terapéutica caracterizaron los enfermos con este signo. 